\title{
CONTROLE EM TRÊS EIXOS PARA AQUISIÇÃO DE ATITUDE POR SATÉLITE UNIVERSITÁRIO PARTINDO DE CONDIÇÕES INICIAIS DESFAVORÁVEIS
}

\author{
Wilder da Vera Cruz Viegas* \\ viegas_wilder@yahoo.com.br \\ Ronaldo Waschburger* \\ ronaldoweita.br
}

\author{
Davi Antônio dos Santos* \\ davistseita.br \\ Jacques Waldmann* \\ jacqueseita.br
}

\author{
${ }^{*}$ Depto. Sistemas e Controle, Divisão de Engenharia Eletrônica \\ Instituto Tecnológico de Aeronáutica - Praça Marechal Eduardo Gomes, 50 - Vila das Acácias \\ CEP 12228-900 - São José dos Campos, SP, Brasil
}

\begin{abstract}
3-axis control of a university satellite for attitude acquisition departing from unfavorable initial conditions The design of a low cost university satellite ITASAT seeks to meet launch constraints as a secondary, piggyback payload. The dual-spin configuration has been investigated to determine the performance of the autonomous Attitude Control System (SCA) when the satellite separates from the launch vehicle in an unfavorable initial condition prior to attitude acquisition. The dual-spinner is controlled in three axes to acquire and maintain a single face pointing to the Sun by means of a momentum wheel whose rotation axis is orthogonal to that face, and a triad of air-core coil magnetotorquers that provide gyroscopic stiffness and control torques to maneuver and point the solar panel to the Sun. The SCA starts actuation as from separation, and operates in closed loop with estimates of the satellite attitude and angular velocity. These estimates are computed by an extended Kalman filter (FKE) that processes vector measurements of the Sun direction and the geomagnetic field provided by Sun sensors and a triaxial magnetometer. Starting from unfavorable initial conditions, approximately 1.5 day has been required to yield a pointing error under $10^{\circ}$, and less than two days to maintain the error below
\end{abstract}

Artigo submetido em 12/12/2010 (Id.: 1232)

Revisado em 11/02/2011, 23/05/2011

Aceito sob recomendação do Editor Associado Prof. Daniel Coutinho $0.1^{\circ}$. Satellite oscillations about the wheel axis have been within an acceptable margin. The duration of the maneuvers in relation to the capacity of the batteries on board have confirmed the feasibility of the dual-spin configuration. The resulting performance and complexity have been compared with those of the rigid-body spin stabilization to select an appropriate configuration for the satellite mission.

KEYWORDS: Artificial satellite, attitude control, extended Kalman filter, magnetometer, torque coils, momentum wheel.

\section{RESUMO}

A concepção de um satélite universitário de baixo custo busca atender as restrições de seu lançamento como carga útil secundária. A configuração girostato foi investigada para avaliar o desempenho do Sistema de Controle de Atitude (SCA) autônomo durante a aquisição de atitude quando o satélite se separa do veículo lançador em uma condição inicial desfavorável. A configuração girostato é controlada em três eixos e busca manter voltada para o Sol a única face com o painel solar. Uma roda de momentum, cujo eixo de rotação é ortogonal àquela face, provê rigidez giroscópica para, em conjunto com três bobinas de torque com núcleo de ar, gerar torques para controle de atitude em três eixos. O SCA inicia sua ação a partir da separação, operando em malha fechada com a estimação da atitude e 
da velocidade angular do satélite. Essas estimativas são fornecidas por um filtro de Kalman estendido (FKE) que processa medidas vetoriais da direção do Sol e do campo geomagnético oriundas de sensores de Sol e magnetômetro triaxial. Partindo de condições desfavoráveis, os resultados mostraram que o apontamento do satélite demanda cerca de um dia e meio dia para prover erro de apontamento inferior a $10^{\circ}$ e menos de dois dias para manter o erro abaixo de $0,1^{\circ}$. Em torno do eixo da roda, ocorre oscilação do satélite em torno da referência, mas dentro de uma margem aceitável. A duração das manobras em relação à capacidade de carga das baterias embarcadas confirmou a viabilidade da configuração girostato. O desempenho e a complexidade dessa configuração foram comparados com a estabilização de corpo rígido com rotação spin visando selecionar configuração apropriada para a missão do satélite.

PALAVRAS-CHAVE: Satélite artificial, controle de atitude, filtro de Kalman estendido, magnetômetro, roda de momentum.

\section{INTRODUÇÃO}

A presente investigação foi inicialmente motivada pelo projeto ITASAT, que envolve a concepção, desenvolvimento, integração, testes, validação, lançamento e operação de um satélite de baixo custo por docentes e discentes universitários sob coordenação do Instituto Tecnológico de Aeronáutica (ITA), com consultoria técnica e infra-estrutura laboratorial do Instituto Nacional de Pesquisas Espaciais (INPE) e patrocínio da Agência Espacial Brasileira (AEB). $\mathrm{O}$ projeto ITASAT enriquece a experiência e contribui para a capacitação de professores e a formação de estudantes de graduação e pós-graduação mediante atuação técnica-científica nos diversos subsistemas embarcados e equipamentos de solo. O projeto objetiva construir um satélite universitário de baixo custo que busque prover uma capacidade operacional relevante, ainda que limitada. Nesse processo, estão sendo produzidos conhecimentos teóricos e aplicados relevantes para o desenvolvimento do setor aeroespacial brasileiro. A carga útil principal do ITASAT consiste num equipamento para coleta de dados ambientais que deverá auxiliar e dar continuidade ao Sistema de Coleta de Dados Ambientais Brasileiro, através do qual são transmitidos para estações terrenas dados ambientais e meteorológicos que foram adquiridos por plataformas de coleta de dados espalhadas pelo território brasileiro.

Projetados no INPE, hoje participam desse sistema os satélites de coleta de dados (SCDs) SCD1 e SCD2, ambos lançados como cargas pagas na década de 1990 e dotados de configuração de corpo rígido estabilizado por rotação em torno do eixo de maior inércia (estabilização por rotação spin). A atitude é controlada por atuação magnética auxiliada por um anel parcialmente preenchido com óleo de silicone para amortecimento passivo de nutação (Carrara e Guedes, 1994; Kuga et al. 1999).

O satélite universitário de baixo custo deverá ser lançado como carga secundária, voando de carona em um veículo lançador. O satélite deverá operar na órbita em que se der a separação da carga principal do veículo lançador. Portanto, o satélite pode estar sujeito a iniciar sua operação sob condições iniciais desfavoráveis de atitude e velocidade angular após a separação do lançador. Esta investigação assumiu como provável condição operacional, por razões que fogem do escopo deste trabalho, uma órbita circular, polar, com inclinação de $98^{\circ} \mathrm{em}$ relação ao plano equatorial, Sol-síncrona - 10:30h AM - e com 750km de altitude.

O satélite deve apontar o painel solar para o Sol com erro inferior a $10^{\circ}$ para recarregar suas baterias embarcadas e apontar suas antenas ao sobrevoar as estação terrenas que recebem os dados coletados pelo satélite. Adicionalmente, o SCA deve ter sua implementação em tempo real viável frente aos sensores, atuadores e os recursos computacionais embarcados, como os do kit Atmel TSC695F empregado no desenvolvimento de um computador de bordo (Atmel, 2011).

Partindo da experiência prévia do INPE e considerando a incerteza de como ocorrerá a separação do satélite, se com rotação spin ou estabilizado em três eixos, uma configuração semelhante aos dos SCDs foi investigada por Waschburger $e t$ al. (2009). Consistiu de estabilização por rotação spin dotada de atuação puramente magnética. Porém, essa configuração foi investigada em órbita baixa, com inclinação de $25^{\circ}$, e um erro inicial de apontamento limitado, da ordem de $15^{\circ}$. Infelizmente, investigação posterior mostrou haver divergência quando há erro inicial maior e capotamento em três eixos após separação na órbita baixa, assim como também ao operar em órbita polar. Adicionalmente, a previsão de geração de potência pelos painéis solares nessa configuração indicou oscilação excessiva e valor insuficiente frente ao consumo esperado.

Operar em órbita polar, com os painéis solares gerando potência adequadamente e partindo de condições iniciais desfavoráveis, demanda configuração distinta. Daí advém a motivação para o girostato com atuação magnética investigado neste trabalho. As duas configurações são então avaliadas de forma comparativa para que uma seja selecionada para implementação de acordo com os objetivos e requisitos definidos para a missão. 


\subsection{Análise, Projeto e Revisões da Missão}

A análise e o projeto de missões espaciais envolvem o refinamento gradual e iterativo dos objetivos da missão, de seus requisitos e restrições e dos métodos para satisfazê-los (Larson e Wertz, 1999). Inicialmente, as necessidades da missão são determinadas em termos qualitativos com base na definição da missão e seus objetivos. A seguir, requisitos são quantificados, condicionados às necessidades da missão, às tecnologias aplicáveis e às restrições, inclusive de custo. Os requisitos quantitativos são reavaliados e revistos ao longo das etapas de concepção e projeto. A definição da missão e seu detalhamento frequentemente envolvem a investigação de configurações e conceitos alternativos, incluindo a seleção da carga útil apropriada, a disponibilização ao usuário final dos dados adquiridos pela carga útil, a programação, o gerenciamento e o controle das diversas fases da missão.

A definição da missão e de seus requisitos é um processo colaborativo entre as equipes envolvidas com a análise e o projeto da missão, os usuários dos serviços a serem prestados pelo satélite e os patrocinadores que arcam com o custo da missão. Os objetivos e as restrições da missão devem ser traduzidos em requisitos de produtos, tanto de software quanto de hardware. Esses requisitos devem ser muito bem definidos para a implementação dos sistemas que compõem o satélite. Os objetivos, restrições e requisitos impactam diretamente na configuração adotada para o satélite e na implementação e integração de seus diversos sistemas.

A definição da missão e seus requisitos são revisados e avaliados na Revisão da Definição da Missão (MDR) e na Revisão Preliminar dos Requisitos (PRR). Essas revisões são conduzidas por comissões de especialistas com profundo conhecimento nas áreas envolvidas. As equipes envolvidas com a análise e o projeto da missão devem prover informações e dados às comissões de forma a apoiá-las no processo de revisão, avaliação e tomada de decisões. Para o ITASAT, as comissões favoreceram metodologias e tecnologias de baixo custo e risco tecnológico aceitável, buscando obter significativa probabilidade de sucesso da missão.

Este artigo focaliza a investigação da configuração girostato e seu Sistema de Controle de Atitude (SCA) partindo de condições iniciais desfavoráveis e compara seu desempenho e complexidade com os da configuração de corpo rígido estabilizado por rotação spin com atuação puramente magnética (Waschburger et al., 2009). Na PRR, essas configurações foram comparadas entre si com vistas a selecionar uma delas à luz da experiência acumulada em projetos anteriores de satélites no INPE.
A estrutura do artigo é como a seguir. A Seção 2 descreve a atuação magnética para satélites de baixo custo. A Seção 3 apresenta a configuração girostato, sensores e atuadores e delineia a estimação de atitude e velocidade angular. A Seção 4 apresenta a modelagem da dinâmica de atitude do satélite. A Seção 5 descreve o controle de atitude. A Seção 6 analisa os resultados das simulações e suas consequências para a tomada de decisões na PRR, em que pese a complexidade decorrente da configuração girostato. Ao final, a Seção 7 apresenta as conclusões da investigação conduzida.

\section{CONTROLE MAGNÉTICO}

Atraente devido ao baixo custo, a estabilização magnética passiva não requer dispêndio de energia. Trata-se da iteração permanente entre o vetor indução do campo geomagnético e atuadores magnéticos passivos, como imãs permanentes e barras magneticamente permeáveis com histerese, que são simples e confiáveis. Por outro lado, o controle magnético ativo é mais complexo e demanda consumo de energia para ativar bobinas de torque, também conhecidas como magnetotorqueadores (MTQs), mas provê maior flexibilidade. O controle magnético ativo é mais efetivo em órbitas de inclinação elevada e torna-se magneticamente limpo quando os MTQs estão desligados (Mesch, 1969), assim atenuando o torque de perturbação magnético e reduzindo os erros de medida do campo geomagnético pelo magnetômetro.

No caso da configuração estabilizada por rotação spin, após a separação em condições iniciais desfavoráveis, os MTQs são rapidamente chaveados na fase de aquisição de atitude e de velocidade de spin. Nessa situação, a constante de tempo no transiente da corrente - dada pela razão entre indutância e resistência da bobina - é característica relevante (Mesch, 1969). Uma vez comandado seu desligamento, o MTQ com núcleo de ar se torna magneticamente limpo passado o transiente de corrente. Já o MTQ de núcleo ferromagnético, para um mesmo valor de dipolo magnético, requer menos potência e é mais leve que o de núcleo de ar (Roth, 2001), mas, quando desligado, age como barra magneticamente permeável que atenua a velocidade angular (Mesch, 1969). Mais recentemente, Carrara (1996) apresentou projeto de MTQ com núcleo ferromagnético, mas sem abordar o transiente de corrente e a magnetização residual do núcleo, que depende do histórico de ativação dos MTQs nas manobras do satélite, e o efeito sobre as medidas do magnetômetro. $\mathrm{O}$ transiente de corrente e a magnetização residual são relevantes nas medições feitas pelo magnetômetro, cujas leituras, conforme investigado aqui, são intercaladas pelo acionamento de MTQs.

No tocante ao controle magnético ativo para satélites rígidos estabilizados por rotação spin, Ergin e Wheeler (1965) 
apresentaram sistema autônomo com um único MTQ para apontamento do eixo de spin, porém assumindo que a rotação spin fosse constante e a nutação, pequena. Conforme explicado mais adiante na Seção 3.3, nutação pequena corresponde ao momentum angular próximo do alinhamento com o eixo de spin. Adicionalmente, verificou-se que se mantém constante o produto do peso com a potência requerida para acionar um MTQ com núcleo de ar, uma vez fixados o dipolo magnético, o material do enrolamento e os diâmetros do fio e da bobina. Portanto, fixada a tensão de alimentação, um MTQ com menos espiras e menor área para obter transiente de corrente mais rápido demandará maior corrente de acionamento para produzir o dipolo magnético desejado.

Ainda assumindo pequena nutação e rotação spin constante, Wheeler (1967) verificou a estabilidade de lei de controle magnético para erros de apontamento de grande magnitude. Rennard (1967), também desconsiderando nutação e assumindo rotação spin constante, investigou o efeito médio do torque de controle magnético devido ao chaveamento do MTQ em instantes pré-programados numa órbita. Ambas as abordagens desconsideraram a necessidade de estimação da atitude do eixo de spin para realizar a lei de controle

Sorensen (1971) tratou do controle magnético com um triedro de MTQs operando em malha fechada com a estimação de atitude e velocidade angular. A estimação usou a modelagem linearizada em torno da rotação spin nominal e reduzida nutação, desconsiderando a interferência da corrente nos MTQs sobre as medidas de magnetômetro.

Shigehara (1972) propôs a ação liga-desliga de dois MTQs com núcleo de ar posicionados ortogonalmente para apontar o eixo de spin, controlar a rotação spin e atenuar a nutação. Os MTQs eram acionados segundo dois critérios distintos, ora visando reduzir o erro quadrático de apontamento do momentum angular, ora o erro quadrático de magnitude decorrente do erro na rotação spin. Esses critérios de acionamento derivaram de duas análises que buscavam, em separado, a estabilidade assintótica em malha fechada. A abordagem assumiu que atitude e velocidade angular eram conhecidas, desconsiderou erros de estimação, de leitura do magnetômetro e a indutância mútua no acionamento simultâneo de MTQs. Não houve prova se o sistema resultante do chaveamento entre os dois critérios para acionamento dos MTQs era assintotica estável (Zhao, 2009).

Lovera et al. (2002) investigaram a síntese de controlador LQR baseado na solução da equação diferencial de Riccatti com dinâmica linearizada periódica da atitude do satélite em órbita, em malha fechada com um filtro de Kalman para estimação da atitude, velocidade angular e perturbações periódicas. Além da atuação magnética em três eixos, empregaram uma roda de momentum e um sensor de estrelas - que é um sensor caro para uso em satélite de baixo custo. Kulkarni e Campbell (2004) projetaram um controlador linear com atuação magnética em três eixos com abordagem H-infinito estendida para tratar a dinâmica linear periodicamente variante no tempo, sem considerar os erros na estimação de atitude. Gravdahl (2004) empregou análise de estabilidade baseada em Lyapunov para síntese de controle magnético não linear de atitude em três eixos, desconsiderando a estimação de atitude. Os sinais de controle em Lovera et al. (1972), Kulkarni e Campbell (2004) e Gravdahl (2004) eram dipolos magnéticos que assumiam valores num intervalo contínuo - o que torna a eletrônica de acionamento mais complexa do que na ação liga-desliga.

\section{A CONFIGURAÇÃO GIROSTATO}

Objetivando que o painel solar gerasse mais potência, sem as flutuações observadas na configuração de corpo rígido estabilizado por rotação spin, foi investigada uma configuração girostato, ou dual-spin (Hughes, 1986; Sidi, 1997). Sua aplicação ao ITASAT foi descrita em Gentina et al. (2009) e aprofundada por Viegas (2010) e Viegas e Waldmann (2010). O satélite tem formato cubóide com dimensões 700x600x400mm com painel solar apenas numa face, a de maior área, sempre voltada para o Sol, conforme visto na Fig.1, e apresenta massa de 85 kilogramas.

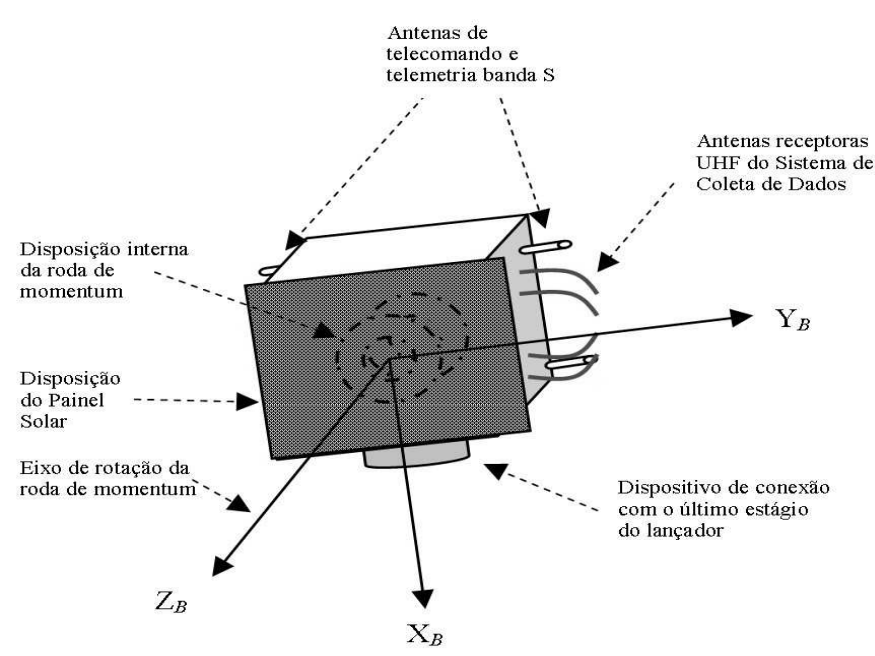

Figura 1: Configuração girostato do satélite universitário.

O girostato possui uma roda de momentum para prover inércia giroscópica e três MTQs com núcleos de ar para controle da atitude em três eixos. O eixo de rotação da roda é perpendicular à face com o painel solar e está alinhado 
com o eixo principal de maior inércia do satélite. Para manter-se voltada para o Sol, essa face deve girar lentamente em torno de um eixo perpendicular ao plano da eclíptica para acompanhar a translação da Terra em torno do Sol durante o ano, a uma taxa de aproximadamente $1 \%$ dia.

Em caso de pane que resulte na parada da roda após a aquisição da atitude, o momentum angular é transmitido ao corpo do satélite para manter o painel solar apontando em direção adequada por algumas semanas. Assim, as baterias continuariam sendo carregadas, quando fora dos eclipses, ainda que a Terra continue deslocando-se ao longo de sua órbita em torno do Sol e o painel solar gradualmente perca seu alinhamento em direção ao Sol. Nesse período de pane da roda, se os enlaces de telemetria e telecomando estiverem funcionais, continuaria a recepção em solo dos dados do satélite, com vistas a diagnosticar a causa da falha e, se possível, tomar as ações cabíveis para retornar o satélite à fase operacional.

\subsection{Sistema de Determinação de Atitude (SDA)}

O SCA emprega um Sistema de Determinação de Atitude (SDA) que estima a atitude e a velocidade angular nos três eixos do satélite com base em medidas vetoriais usando sensores de Sol e um magnetômetro triaxial, conforme mostra a Fig.2 (Santos, 2008, 2009). Logo após a separação e previamente à estimação de atitude e velocidade angular, é fechada apenas a chave $\mathrm{CH}_{1}$ para começar a estimação dos biases residuais nas medidas do magnetômetro tri-axial, indicado por SM, por um filtro de Kalman estendido (FKE), designado por $\mathrm{E}_{1}$. Após a convergência de $\mathrm{E}_{1}$, subtraem-se os biases estimados da medida vetorial bruta $\breve{b}_{1}$ do magnetômetro na etapa de pré-processamento $\mathrm{PP}_{1}$, e resultam as medidas calibradas $\mathbf{b}_{\mathbf{1}}$ do campo geomagnético.

A etapa de pré-processamento PP2 seleciona o sensor de Sol dentre os três embarcados, $\mathrm{SS}_{j}, j=1,2,3$, converte os ângulos que medem a direção do Sol em uma pseudomedida vetorial da direção do Sol, com magnitude unitária, e passa sua representação do sistema de coordenadas do sensor para o sistema de coordenadas do corpo do satélite. Assumiu-se que, fora do eclipse, o Sol está sempre no campo de visão de, pelo menos, um dos sensores de Sol.

Após a convergência de $\mathrm{E}_{1}$, fecha-se a chave $\mathrm{CH}_{2}$ e outro FKE, para estimação de atitude e velocidade angular, designado por $\mathrm{E}_{2}$, passa a processar as medidas dos vetores da direção do Sol e do campo geomagnético calibrado. Esse FKE relaciona, por meio de uma equação de medidas, os vetores medidos e representados no sistema do corpo com os mesmos vetores, de referência, representados no sistema geocêntrico inercial. Os vetores de referência são computados com os modelos embarcados $\mathrm{M}_{1}$ e $\mathrm{M}_{2}$ da mecânica celeste e do campo geomagnético, respectivamente, assim como um modelo da cinemática orbital do satélite que estima sua posição.

Os modelos embarcados são simplificados para não sobrecarregarem os recursos computacionais disponíveis a bordo, mas são adequadamente acurados para a finalidade de estimação de atitude e velocidade angular do satélite. O modelo embarcado $\mathrm{M}_{1}$ é o WMM 2005 que descreve o campo magnético e é limitado a ordem 4 na expansão em série de harmônicos esféricos (McLean et al., 2004). Já o modelo embarcado $\mathrm{M}_{2}$ descreve o movimento do satélite em uma órbita Kepleriana, considerando o coeficiente $\mathrm{J}_{2}$ que caracteriza a forma oblata da Terra, i.e., achatada ao longo do seu eixo de rotação (Vallado et al., 2006). Os parâmetros usados nos modelos embarcados são periodicamente atualizados pela estação de controle da missão em solo (vide Fig.6).

A frequência de amostragem do magnetômetro e do sensor de Sol é $10 \mathrm{~Hz}$, obtida da sintonia do FKE para estabilização por rotação spin em órbita de baixa inclinação (Santos, 2008; Santos e Waldmann, 2009). Aqui, o FKE E 2 para estimação de atitude e velocidade angular foi alterado para incluir a dinâmica da roda de momentum e requereu nova sintonia, mas manteve-se a frequência de amostragem de $10 \mathrm{~Hz}$.

\subsection{Magnetotorqueadores (MTQs)}

Após a separação, devido ao capotamento inicial do satélite, as medidas nos três eixos do magnetômetro podem variar significativamente e os MTQs precisam ser rápida e acuradamente chaveados para adquirir a atitude requerida sem excitar o movimento de nutação. A configuração investigada adotou MTQs com núcleo de ar para reduzir a indutância e, por conseguinte, o transiente de corrente no caso de acionamento por um degrau de tensão. Portanto, o torque de controle resultante é conhecido para uso pelo SDA.

A matriz de covariância do erro de modelagem no FKE $\mathrm{E}_{2}$ para estimação de atitude e velocidade angular foi sintonizada considerando que o sinal de controle para cada MTQ é uma corrente de acionamento com magnitude fixa e conhecida (Shigehara, 1972; Santos, 2008; Santos e Waldmann, 2009). O ciclo liga-desliga do MTQ a $10 \mathrm{~Hz}$ mantém-se $80 \mathrm{~ms}$ ativo e $20 \mathrm{~ms}$ inativo. Uma tríade de MTQs, ortogonais entre si e alinhados com o sistema do corpo, é empregada.

A polaridade da corrente varia de acordo com a direção requerida para o torque de controle. $\mathrm{O}$ transiente de corrente 


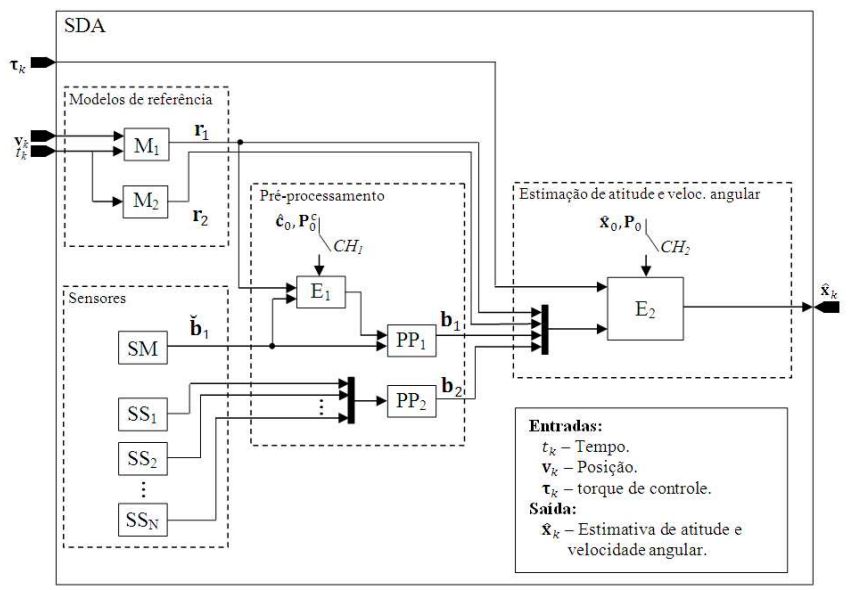

Figura 2: Diagrama de blocos do SDA (Santos, 2008, 2009).

deve ser negligível em relação aos $20 \mathrm{~ms}$ de inatividade, ao fim dos quais as medidas do magnetômetro são lidas (Waschburger et al., 2009). Apenas um MTQ é acionado por vez para eliminar perturbações devido à indutância mútua, cuja modelagem foi desconsiderada no $\mathrm{FKE}_{2}$.

Cada MTQ emprega fio AWG-30, com resistência elétrica por unidade de comprimento $0,351 \Omega / \mathrm{m}$ a $20^{\circ}$ Celsius, comprimento $798 \mathrm{~m}$ e diâmetro médio $0,514 \mathrm{~m}$, com $N=512$ espiras dispostas em 32 camadas e 16 espiras por camada, área de cada espira $A=0,198 \mathrm{~m}^{2}$ e o fio usado no enrolamento com massa de $0,367 \mathrm{~kg}$. Cada MTQ pode gerar um dipolo magnético de magnitude $10,1 \mathrm{~A} . \mathrm{m}^{2}$ com uma corrente $i=0,1 A$ e apresenta constante de tempo estimada em $1,14 \mathrm{~ms}$. Alimentado com 28VDC, um MTQ consome $2,8 \mathrm{~W}$.

\subsection{Nutação e Amortecimento Magnético}

O satélite é liberado em órbita com velocidade angular (capotamento, ou tumbling), a roda travada em relação ao corpo do satélite e o erro de apontamento inicial de aproximadamente $180^{\circ}$, ou seja, a face com painel solar apontando na direção oposta àquela desejada.

Essa condição inicial difere significativamente dos lançamentos de ambos os satélites SCDs como cargas principais. Por isso, os SCDs foram colocados em órbita já estabilizados por rotação spin e com o apontamento muito próximo do correto. Cada SCD é equipado com um anel de amortecimento de nutação parcialmente preenchido com fluido, instalado ortogonalmente ao eixo de maior inércia e excentricamente posicionado em relação ao referido eixo (Alfriend, 1974; Lopes, 1987; da Fonseca e de Souza, 1990). Devido à rotação spin em torno do eixo de maior inércia, o fluido no anel de amortecimento se mantém como um bloco na região do anel mais distante do eixo de rotação.
A nutação, vista na Fig. 5, consiste no desalinhamento $\delta_{\text {nut }}$ entre o momentum angular $\mathbf{H}$ e o eixo de spin designado por $\mathbf{k}_{b}$, produzindo oscilações em torno dos eixos ortogonais ao eixo de spin. A nutação deve ser atenuada, mas pode ser excitada pelo acionamento dos MTQs. O bloco de fluido, oscilando no interior do tubo, sofre atrito ao mover-se relativo à parede interior do tubo. Isso resulta em um sorvedouro de energia cinética de rotação e provê amortecimento passivo da nutação.

Devido ao capotamento inicial na separação do satélite universitário, diferentemente dos SCDs, o SCA aqui investigado não envolve $\mathrm{o}$ anel de amortecimento para evitar a ocorrência de fluido esparramado movimentando-se no interior do anel (sloshing). Ainda que se possa aventar a hipótese de que o impacto do sloshing seja negligível, a validação dessa hipótese demandaria modelar o comportamento dinâmico do fluido nessa condição (Vreeburg, 2005). Para reduzir a complexidade do projeto, optou-se por amortecimento ativo da nutação por atuação puramente magnética que, para não excitar a nutação, deve ligar e desligar os MTQs rápida e acuradamente, quando necessário (Shigehara, 1972).

\subsection{Autonomia do Sistema de Controle de Atitude}

Em abordagens anteriores, com o fito de alcançar maior simplicidade e confiabilidade, as medidas dos sensores de Sol e magnetômetro eram enviadas via enlace de telemetria para estimação da atitude na estação de controle em solo e, quando o satélite estivesse novamente com linha de visada para a estação em solo com o enlace de telecomandos ativo, ocorria o upload do cronograma de acionamento dos MTQs para armazenamento no computador de bordo (Rennard, 1967; Carrara e Guedes, 1994; Lopes et al., 1994; Kuga et al., 1999).

Aqui, o SCA é autônomo para, após a separação, iniciar a aquisição da atitude requerida e evitar descarga demasiada das baterias embarcadas. O início da aquisição de atitude independe de haver enlace de dados e telecomandos ativo. As fases de controle de atitude serão monitoradas quando o enlace estiver ativo e, se necessário, modificadas pelo controle da missão.

\section{MODELAGEM DA DINÂMICA DA ATITUDE}

Os sistemas de coordenadas cartesianas geocêntrico inercial $\mathbf{S}_{I}$, orbital $\mathbf{S}_{o}$, e do corpo do satélite $\mathbf{S}_{b}$ que descrevem a atitude do satélite e seu movimento ao longo da órbita, estão na Fig.3. $\mathbf{R}_{s}$ é o vetor posição do centro do satélite 
em relação ao centro da Terra; $u$ é argumento da latitude do satélite com relação ao nodo ascendente na sua órbita circular; $\Omega$ é a ascensão reta do nodo ascendente; e $i$ a inclinação da órbita.

$\mathrm{O}$ sistema de referência inercial $\mathbf{S}_{I}$ não gira e tem origem no centro da Terra. $\mathrm{O}$ eixo inercial $\mathbf{i}_{i}$ aponta para o equinócio vernal, enquanto $\mathbf{k}_{i}$ é ortogonal ao plano equatorial, portanto alinhado com o eixo de rotação da Terra. O eixo orbital $\mathbf{i}_{o}$ aponta para o nodo ascendente e $\mathbf{k}_{o}$ é perpendicular ao plano da órbita. Os ângulos de Euler que parametrizam a atitude de $\mathbf{S}_{b}$ em relação ao sistema de coordenadas da órbita $\mathbf{S}_{o}$ são de acordo com a seqüência de rotações: $\psi$ (azimute) em torno de $\mathbf{k}_{o}, \theta$ (elevação) em torno de $\mathbf{j}_{b}$ e $\phi$ (rotação) em torno de $\mathbf{k}_{b}$.

O modelo da dinâmica de atitude do satélite em órbita, na configuração girostato e sujeito a torques externos, é dado pela Eq. (1) e pela Eq. (2).

$$
\begin{gathered}
\stackrel{b}{\boldsymbol{\omega}}=\mathbf{I}_{A}^{-1}\left[\left(\mathbf{I}_{s} \boldsymbol{\omega}\right) \times \boldsymbol{\omega}+\left(\mathbf{I}_{w} \boldsymbol{\Omega}_{w}\right) \times \boldsymbol{\omega}+\mathbf{T}-\mathbf{u}_{w}\right] \\
\stackrel{b}{\boldsymbol{\Omega}_{w}}=\mathbf{I}_{w}^{-1}\left[-\mathbf{I}_{w B} \stackrel{b}{\boldsymbol{\omega}}+\mathbf{u}_{w}\right] \\
\mathbf{I}_{w}=\mathbf{I}_{w A}+\mathbf{I}_{w B} ; \quad \mathbf{I}_{w A}=\left[\begin{array}{lll}
\mathrm{I}_{w 1} & 0 & 0 \\
0 & \mathrm{I}_{w 2} & 0 \\
0 & 0 & 0
\end{array}\right] ; \\
\mathbf{I}_{w B}=\left[\begin{array}{lll}
0 & 0 & 0 \\
0 & 0 & 0 \\
0 & 0 & \mathrm{I}_{w 3}
\end{array}\right] ; \quad \mathbf{I}_{A}=\mathbf{I}+\mathbf{I}_{w A} ; \quad \mathbf{I}_{s}=\mathbf{I}+\mathbf{I}_{w} .
\end{gathered}
$$

sendo que I é a matriz de inércia do satélite sem a roda, $\mathbf{I}_{w}$ é a matriz de inércia da roda; $\boldsymbol{\omega}=\left[\begin{array}{lll}\omega_{1} & \omega_{2} & \omega_{3}\end{array}\right]^{T}$ e $\boldsymbol{\Omega}_{w}=$ $\left[\begin{array}{lll}0 & 0 & \Omega_{w}\end{array}\right]^{T}$ são, respectivamente, o vetor velocidade angular do satélite com relação ao sistema inercial $\mathbf{S}_{I}$ e o vetor velocidade angular da roda de momentum com relação ao satélite. Ambos esses vetores são representados no sistema de do corpo $\mathbf{S}_{b} ; \stackrel{b}{\boldsymbol{\omega}}$ e $\stackrel{b}{\boldsymbol{\Omega}_{w}}$ são, respectivamente, as derivadas temporais do vetor velocidade angular do satélite e do da roda segundo um observador fixo ao corpo; $\mathbf{T}$ é o somatório de torques externos agindo sobre o sistema e $\mathbf{u}_{w}=\left[\begin{array}{lll}0 & 0 & u_{w}\end{array}\right]^{T}$ é o torque interno que o motor, fixo ao corpo do satélite, exerce sobre a roda de momentum.

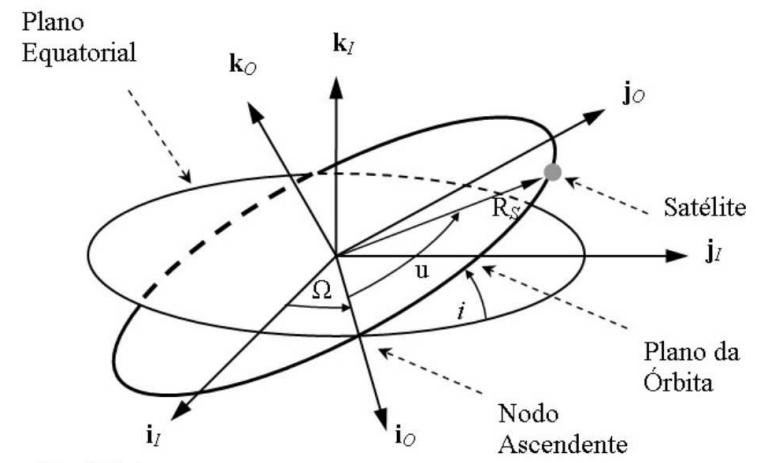

Equinócio

Vernal

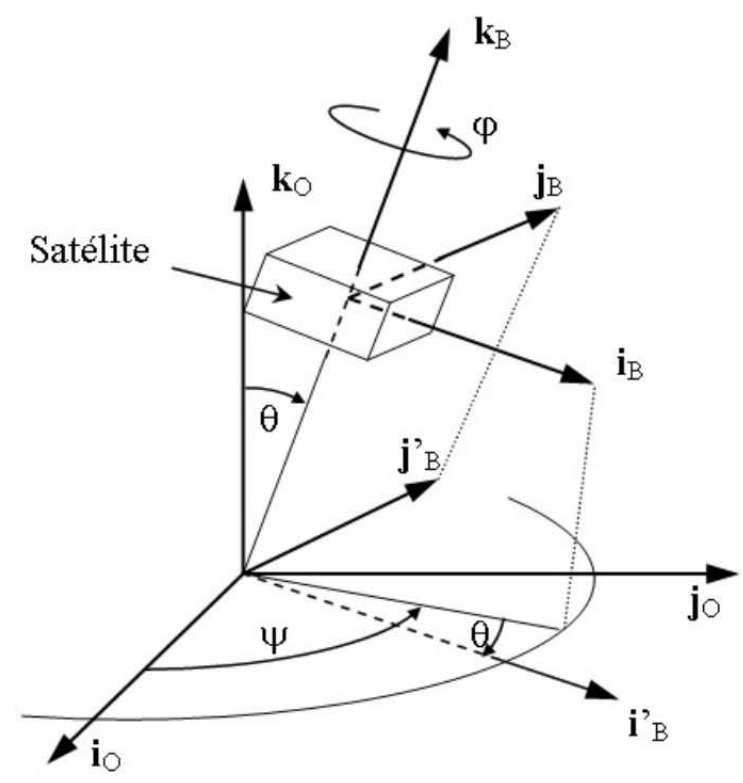

Figura 3: Sistemas de coordenadas cartesianas inercial geocêntrico $S_{I}$, orbital $S_{o}$ e do corpo $S_{b}$ (Shigehara, 1972).

O triedro de MTQs é alinhado com os eixos $\mathbf{i}_{b}, \mathbf{j}_{b}$ e $\mathbf{k}_{b}$, que coincidem com os eixos principais. Foi assumido que o torque interno dispendido em atrito na roda é negligenciável frente aos demais efeitos e, portanto, que a corrente de acionamento do motor da roda é integralmente transformada em torque que gera aceleração angular da roda.

Para evitar singularidades, a cinemática da atitude do satélite é descrita usando-se quatérnion, conforme a Eq. (4).

$$
\dot{\mathbf{q}}=\left[\begin{array}{c}
\dot{\lambda} \\
\dot{q}_{1} \\
\dot{q}_{2} \\
\dot{q}_{3}
\end{array}\right]=\frac{1}{2}\left[\begin{array}{llll}
0 & -\omega_{1} & -\omega_{2} & -\omega_{3} \\
\omega_{1} & 0 & \omega_{3} & -\omega_{2} \\
\omega_{2} & -\omega_{3} & 0 & \omega_{1} \\
\omega_{3} & \omega_{2} & -\omega_{1} & 0
\end{array}\right] \cdot\left[\begin{array}{l}
\lambda \\
q_{1} \\
q_{2} \\
q_{3}
\end{array}\right]
$$


em que $\mathbf{q}=\left[\begin{array}{llll}\lambda & q_{1} & q_{2} & q_{3}\end{array}\right]^{T}$ representa o quatérnion de rotação que leva do sistema inercial geocêntrico $\mathbf{S}_{I}$ ao sistema do corpo $\mathbf{S}_{b}$. O controle magnético é dado pelo torque externo $\mathbf{T}_{M a g}$ resultante da interação entre o dipolo magnético $\mathbf{M}$ de um MTQ e o campo geomagnético B, conforme a Eq. (5).

$$
\mathbf{T}_{M a g}=\mathbf{M} \times \mathbf{B}
$$

$\mathrm{O}$ torque de perturbação magnética $\mathbf{T}_{E d d y}$ devido a correntes induzidas no corpo do satélite ao girar no campo geomagnético é modelado pela Eq. (6).

$$
\mathbf{T}_{E d d y}=k_{e}(\omega \times \mathbf{B}) \times \mathbf{B}
$$

em que o coeficiente $k_{e}$ depende da geometria e da condutividade do satélite (Kuga et al., 1987).

O torque de perturbação $\mathbf{T}_{G G}$ causado pelo gradiente de gravidade sobre o corpo do satélite é modelado pela Eq. (7) (Wertz, 1978):

$$
\mathbf{T}_{G G}=\left(3 \mu /\left\|\mathbf{R}_{s}\right\|^{3}\right) \cdot\left[\hat{\mathbf{R}}_{s} \times\left(\mathbf{I}_{\mathbf{S}} \cdot \hat{\mathbf{R}}_{s}\right)\right]
$$

em que $\mathbf{I}_{s}$ é a matriz de inércia, $\mu$ é a constante gravitacional da Terra e $\hat{\mathbf{R}}_{s}$ é o vetor unitário ao longo de $\mathbf{R}_{s}$.

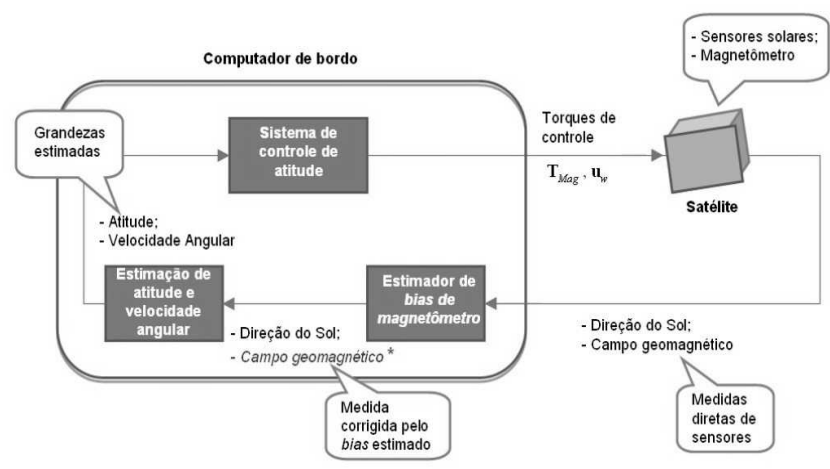

Figura 4: Diagrama de blocos do SCA.

\section{SISTEMA DE CONTROLE DE ATITUDE (SCA)}

O SCA, mostrado na Fig.4, parte de uma condição inicial com um erro de apontamento inicial de $180^{\circ}$ em relação à direção desejada, capotando lentamente, sendo a roda ativada após a separação do satélite. O controle é iniciado após ter convergido a estimação do bias de magnetômetro e sido feita sua calibração (Alonso e Shuster, 2002; Crassidis et al., 2005; Santos, 2009). O controle de atitude prevê três fases de operação que serão descritas a seguir.

\subsection{FASE l:}

Consiste em, imediatamente após a separação do satélite, destravar e acionar o motor da roda de momentum. A aceleração angular da roda é constante devido ao torque constante de $0,02 \mathrm{~N} \cdot \mathrm{m}$ exercido pelo motor da roda, até que atinja sua velocidade nominal de operação de 450RPM em relação ao corpo do satélite. Nessa fase, a ação dos MTQs encontra-se desabilitada. O torque de reação do motor da roda sobre o satélite produz rotação do corpo do satélite em direção inversa àquela da roda. Os torques externos de perturbação são pequenos e é praticamente nula a variação do momentum angular total do sistema composto pelo corpo do satélite e a roda. Devido a produtos de inércia não nulos, o capotamento se acentua com a aceleração angular da roda.

\subsection{FASE II:}

Atingida a velocidade nominal de operação da roda na Fase I, o acionamento do motor da roda é desabilitado e imediatamente acionado o controle magnético com os MTQs. Consiste em, simultaneamente, eliminar o erro angular de apontamento $E_{a p}$ do vetor momentum angular $\mathbf{H}$ e o ângulo de nutação $\delta_{\text {nut }}$ vistos na Fig.5. O erro $E_{a p}$ é dado por:

$$
E_{a p}=\cos ^{-1}\left(\frac{\left\langle\mathbf{H}_{r e f}, \mathbf{H}\right\rangle}{\left\|\mathbf{H}_{r e f}\right\| \cdot\|\mathbf{H}\|}\right)
$$

Em torno dos eixos ortogonais ao eixo da roda, é reduzida a rotação do corpo do satélite para um valor próximo de zero através do torque de controle magnético, que modifica o momentum angular total do satélite com a roda.

O projeto do controle magnético baseou-se na investigação da configuração de corpo rígido estabilizada por rotação spin (Waschburger et al., 2009), cuja lei de controle inspirou-se em Shigehara (1972). Este propôs prova de estabilidade assintótica assumindo satélite idealmente rígido, axissimétrico e estabilizado por rotação spin, considerando individualmente dois critérios para ligar e desligar MTQs - o critério de apontamento do momentum angular e o de controle da velocidade de spin - e chaveando entre os critérios à medida que se desenvolva a aquisição de atitude. 


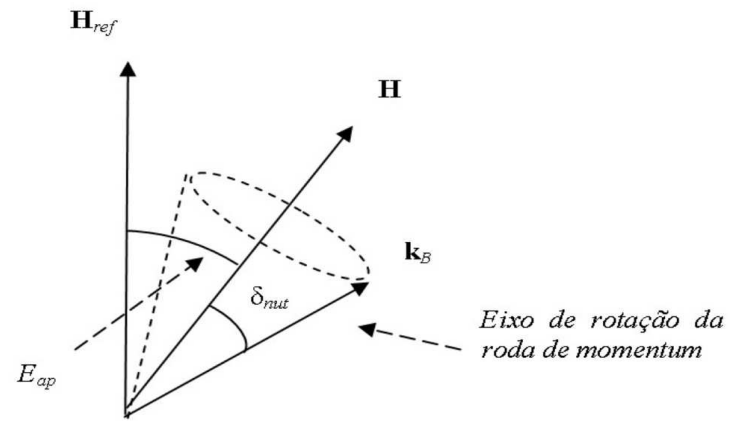

Figura 5: Definição do erro de apontamento e do ângulo de nutação.

De forma geral, um sistema chaveado não herda as propriedades de estabilidade dos subsistemas individuais (Zhao, 2009). Waschburger et al. (2009) empregou um único critério para ligar-desligar um MTQ por vez, considerando conjuntamente o erro de apontamento do momentum angular e o de velocidade de spin, mostrando-se viável na configuração de corpo rígido somente para pequenos erros iniciais de apontamento e em órbita baixa.

Na montagem e integração do satélite, ocorrem diferenças entre as inércias em torno dos eixos principais ortogonais ao eixo de spin, que é o de maior inércia. Em um corpo rígido, o eixo de menor inércia também é um eixo de rotação estável (Hughes, 1986), podendo resultar um ângulo de nutação $\delta_{\text {nut }}$ com magnitude $90^{\circ}$, o que não é aceitável. Aqui, o controle magnético proposto por Waschburger et al. (2009) foi empregado na configuração girostato operando a partir de condições iniciais desfavoráveis após a separação em órbita polar para apontar o momentum angular e, consequentemente, o painel solar, enquanto é também atenuada a nutação.

A ativação dos MTQs busca maximizar a taxa de decaimento da norma-2 do vetor $\mathbf{E}$ de erro de momentum angular, definido como:

$$
\mathbf{E}=\mathbf{H}_{r e f}-\mathbf{H}
$$

entre o momentum angular de referência $\mathbf{H}_{\text {ref }}$ e o momentum angular $\mathbf{H}$ do satélite, incluindo a roda de momentum. O momentum angular de referência $\boldsymbol{H}_{r e f}$ é adotado aqui como sendo $10 \mathrm{~N} \cdot \mathrm{m} \cdot \mathrm{s}$ na direção do Sol:

$$
\mathbf{H}_{r e f}=10 \hat{\mathbf{S}}_{d i r}
$$

em que $\hat{\mathbf{S}}_{d i r}$ é o vetor unitário na direção do Sol a partir do satélite. O valor adotado na Eq. (10) é a metade do utilizado por Waschburger et al.(2009) para aumentar a manobrabilidade e tornar o apontamento mais ágil. $\mathrm{Na}$ configuração girostato, o vetor $\mathbf{H}_{r e f}$ não é constante e varia conforme a Terra se movimenta durante o ano ao redor do Sol a uma taxa de aproximadamente $1 \%$ dia. Assume-se na derivação da lei de controle que $\mathbf{H}_{r e f}$ permanece constante. $\mathrm{Na}$ implementação, entretanto, a direção do Sol e $\mathbf{H}_{r e f}$ são atualizados a cada 5 segundos.

Seja $\boldsymbol{e}_{B}$ o vetor unitário associado a um MTQ cujo dipolo magnético é alinhado com um eixo principal do corpo, $\mathbf{e}_{B}=$ $\left\{\mathbf{i}_{B}, \mathbf{j}_{B}, \mathbf{k}_{B}\right\}$. Então, ativa-se o MTQ cujo eixo $\mathbf{e}_{B}^{*}$ é:

$$
\mathbf{e}_{\mathbf{B}}^{*}=\underset{\mathbf{e}_{\mathbf{B}}}{\arg \max }\left|\hat{\mathbf{E}} \cdot\left(\mathbf{e}_{\mathbf{B}} \times \mathbf{B}_{\mathbf{m}}\right)\right|
$$

em que $\hat{\mathbf{E}}$ é a estimativa do erro de momentum angular na Eq.(9) e $\mathbf{B}_{m}$ é a medida feita pelo magnetômetro. A corrente de acionamento $i$ assume a magnitude máxima no MTQ que satisfizer a Eq.(11), sendo sua polaridade tal que $\mathrm{d}(\hat{\mathbf{E}} \cdot \hat{\mathbf{E}}) / d t<0$. Em um MTQ com núcleo de ar, resulta o dipolo magnético:

$$
\mathbf{M}=N . i . A \mathbf{e}_{\mathbf{B}}^{*}=\beta \mathbf{e}_{\mathbf{B}}^{*} \cdot \operatorname{sgn}\left(\mathbf{E} \cdot\left(\mathbf{e}_{\mathbf{B}}^{*} \times \mathbf{B}\right)\right)
$$

sendo $N$ o número de espiras, $A$ a área de uma espira, $\beta$ a magnitude máxima do dipolo magnético e $\operatorname{sgn}($.) a função sinal. A Eq.5 indica o torque de controle magnético $\mathbf{T}_{M a g}$ que age no satélite. A duração da Fase II foi determinada por simulação.

\subsection{FASE III:}

Adquirido o apontamento no final da Fase II, estabiliza-se a atitude do satélite em torno do eixo de rotação da roda através do acionamento do motor da roda com o controle PD na Eq.13:

$u_{w \_c}=-K_{p}\left(\varphi_{r e f}-\varphi\right)-K_{d}\left(\omega_{3 \_r e f}-\omega_{3}\right), \quad K_{p}, K_{d}>0$

em que $K_{p}$ e $K_{d}$ são ganhos constantes de controle, $u_{w_{-} c}$ é o torque de controle computado da roda, $\varphi$ é a rotação do satélite em torno do eixo da roda $\boldsymbol{k}_{b}$ visto na Fig.3, e $\omega_{3}$ é a velocidade angular do satélite em torno de $\boldsymbol{k}_{b}$.

$\varphi_{\text {ref }}$ e $\omega_{3 \_r e f}$ são os valores de referência para atitude e velocidade angular do satélite, respectivamente, em torno de $\boldsymbol{k}_{b} \cdot \varphi_{\text {ref }}=-90^{\circ}$ de forma que o eixo $\boldsymbol{i}_{b}$ permaneça no plano da órbita e atenda requisito de posicionamento das antenas do satélite (Gentina et al., 2009). Para o satélite 
Tabela 1: Parâmetros de Simulação

Matriz de inércia no modelo:
$\left[\begin{array}{lll}6,5 & 0 & 0 \\ 0 & 6,5 & 0 \\ 0 & 0 & 8\end{array}\right] \mathrm{kg} \cdot \mathrm{m}^{2} \quad(1)$
Matriz de inércia verdadeira:
$\left[\begin{array}{lll}6,5080 & -0,0080 & -0,0080 \\ -0,0080 & 6,4920 & 0,0080 \\ -0,0080 & 0,0080 & 8,0080\end{array}\right] \mathrm{Kg} \cdot \mathrm{m}^{2} \quad(2)$

Atitude de referência em torno do eixo de rotação:

$\varphi_{\text {ref }}=-90^{\circ} ; \omega_{3 \_r e f}=0^{\circ} / s ; \Omega_{w_{-} r e f}=450 \mathrm{RPM}$

Magnitude máxima dos dipolos magnéticos dos MTQs:

Modelo: $\beta=10$ A.m ${ }^{2}(1)$

Verdadeiro: $\beta=10$ A.m ${ }^{2}+/-1$ A.m ${ }^{2}{ }^{(2)}$

Coefi ciente de torque devido à corrente induzida:

$\mathrm{K}_{e}=500 \Omega / \mathrm{m}^{4}$ (Wertz, 1978)

Inclinação da órbita: $\mathrm{i}=98^{\circ}$

Altitude da órbita: $\mathrm{h}=750 \mathrm{~km}$

Argumento da latitude inicial medida do nodo ascendente: $\mathrm{u}_{0}=$ $0^{\circ}$

Ascensão reta inicial do nodo ascendente: $\Omega_{0}=320^{\circ}$

Excentricidade da órbita:

Modelo: $e=0^{(1)}$

Verdadeira: e $=0,001$ (2)

Erro de apontamento inicial: $E_{a p}=180^{\circ} ; s_{\varphi}=-15^{\circ} ; s_{\theta}=$ $180^{\circ}$

Data de lançamento: 01/01/2009; 00h:00min:00s

Covariâncias dos erros de medidas no FKE:

$\mathbf{R}_{m}=4.10^{-14} . \mathbf{I}_{3} \mathrm{~T}^{2}$;

$\mathbf{R}_{s}=\mathbf{D}_{b}^{s}\left[(0,5 \cdot \pi / 180)^{2} \cdot \Pi \cdot \mathbf{I}_{3} \cdot \Pi^{T}+10^{-6} \cdot I_{3}\right] \cdot \mathbf{D}_{b}^{s T} r d^{2} ;$

(3)

$\mathrm{R}_{\Omega_{\mathrm{w}}}=(0,5 \cdot \pi / 180)^{2}(\mathrm{rd} / \mathrm{s})^{2}$

Intensidade do ruído de modelo no FKE (Santos, 2008; Viegas, 2010):

$\mathbf{G Q G}^{T}=\left[\begin{array}{lll}8 \mathbf{I}_{4}\left(s^{-1}\right) & \mathbf{0}_{4 \times 3} & \mathbf{0}_{4 \times 1} \\ \mathbf{0}_{3 \times 4} & 2 \cdot 10^{3} \mathbf{I}_{3}\left(r d^{2} / s^{3}\right) & \mathbf{0}_{3 \times 1} \\ \mathbf{0}_{1 \times 4} & \mathbf{0}_{1 \times 3} & 2 \cdot 10^{5}\left(r d^{2} / s^{3}\right)\end{array}\right]$

Estado inicial no FKE (quatérnion e velocidade angular):

$\hat{\mathbf{x}}_{0 \mid 0}^{*}=[0,39700,69310,5001-0,3345 \ldots$

$1,5 \cdot \pi \mathrm{pi} / 180 \mathrm{rd} / \mathrm{s} 1,5 \cdot \pi / 180 \mathrm{rd} / \mathrm{s} 1,5 \cdot \pi / 180 \mathrm{rd} / \mathrm{s} 0 \mathrm{rd} / \mathrm{s}]^{T}$

Velocidade angular inicial do satélite:

$[1,7 \cdot \pi / 180 \mathrm{rd} / \mathrm{s} 1,7 \cdot \pi / 180 \mathrm{rd} / \mathrm{s} 1,7 \cdot \pi / 180 \mathrm{rd} / \mathrm{s}]^{T}$

Roda de momentum em forma de anel: ${ }^{(4)}$

Diâmetro médio (D): 0,35m

Altura: $0,12 \mathrm{~m}$

Massa (M): $7 \mathrm{~kg}$

Momentos de Inércia: $\mathrm{I}_{w 1}=\mathrm{I}_{w 2}=1 / 8 \mathrm{M} / \mathrm{D}^{2} ; \mathrm{I}_{w 3}=1 / 4 \mathrm{MD}^{2}$

Torque máximo do motor da roda: $0,2 \mathrm{~N} . \mathrm{m}$

$K_{p}=0,01 \mathrm{~A} / \mathrm{rd}$ e $K_{d}=0,15 \mathrm{~A} \cdot \mathrm{s} / \mathrm{rd}$

* A presente tabela foi adaptada de Waschburger et al. (2009).

(1) Parâmetros usados nos modelos simplifi cados embarcados no SDA

(2) Parâmetros verdadeiros para modelos mais elaborados que descrevem a dinâmica de atitude do satélite e torques de perturbação.

(3) $\quad \Pi=\left[\begin{array}{ll}-\sin \left(s_{\varphi}\right) \sin \left(s_{\theta}\right) & \cos \left(s_{\varphi}\right) \cos \left(s_{\theta}\right) \\ -\sin \left(s_{\varphi}\right) \cos \left(s_{\theta}\right) & -\cos \left(s_{\varphi}\right) \sin \left(s_{\theta}\right) \\ \cos \left(s_{\varphi}\right) & 0\end{array}\right]$

(4) $\mathrm{O}$ atrito na roda foi negligenciado. estabilizado em três eixos, a velocidade angular de referência é $\omega_{3 \_r e f}=0^{\circ} / \mathrm{s}$. Portanto, na Fase III busca-se eliminar completamente a rotação do corpo do satélite em torno do eixo da roda.

\section{RESULTADOS E REVISÃO PRELIMINAR DE REQUISITOS (PRR)}

Na Tab.1 são descritos os parâmetros do satélite universitário e da órbita. O desempenho do SCA foi avaliado em termos do erro angular de apontamento $E_{a p}$ do momentum angular, dos ângulos solares $s_{\varphi}$ e $s_{\theta}$, que indicam o desalinhamento entre a normal ao painel solar e a direção do Sol, e da atitude $\varphi$ em torno do eixo de rotação. Os ângulos solares devem ser mantidos em zero para o correto apontamento do painel solar em direção ao Sol.

\subsection{SIMULAÇÃO}

A Fig.6 indica como foi validado o projeto do SCA mediante simulação com Simulink/Matlab. O objetivo é verificar o desempenho do SCA ao interagir com modelos mais realistas das condições ambientais e do comportamento real do satélite. Esses modelos mais realistas compõem o modelo de validação (ground-truth model).

Os componentes do SCA são vistos no bloco "Sistema embarcado". As funções nesse bloco são as que serão executadas no computador de bordo. Os modelos embarcados de referência são os modelos simplificados $\mathbf{M}_{1}$ e $\mathrm{M}_{2}$ descritos na Seção 3.1.

O bloco "Estação base" apresenta os modelos de validação da órbita e do campo geomagnético que são empregados tanto para simular o campo gravitacional e o campo geomagnético a que está sujeito o satélite, quanto para atualizar parâmetros dos modelos embarcados $\mathrm{M}_{1}$ e $\mathrm{M}_{2}$ quando há linha de visada para a estação de controle em solo. No bloco "Estação base", o modelo de órbita é o SGP4 (Vallado et al., 2006) e o de campo magnético, o WMM 2005 de ordem 12 (MacLean $e t$ $a l .$, 2004). Com o campo geomagnético e a direção do Sol computados pelos seus respectivos modelos de validação, são simuladas as medidas dos sensores. Essas medidas simuladas são empregadas nos estimadores e a lei de controle computa o sinal de acionamento para os MTQs e para o motor da roda.

Os blocos "Satélite", "Sensores" e "Atuadores" são modelos de validação, mais complexos que os empregados no projeto do SCA, que buscam descrever de forma mais realista o comportamento dinâmico da atitude do satélite, seus sensores e atuadores, respectivamente. 


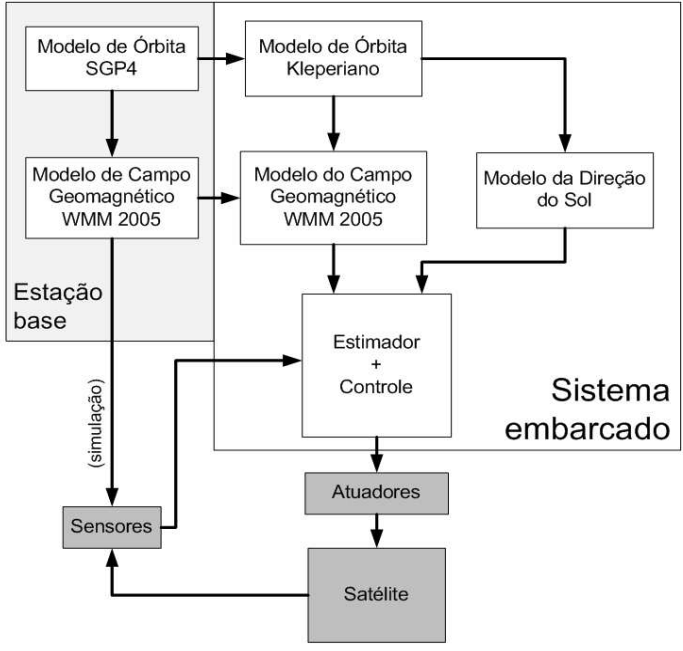

Figura 6: Modelo de simulação (Godoi e Waldmann, 2010)

\subsection{DESEMPENHO DO GIROSTATO}

A Fig.7 e a Fig.8 mostram, respectivamente, a velocidade angular da roda de momentum e o componente da velocidade angular do satélite em torno do eixo da roda na Fase I, que tem duração aproximada de 500s. A velocidade angular em regime da roda se mantém em 450RPM mesmo durante a Fase II, quando o controle da roda é desabilitado, pois foi assumido que a roda não apresenta atrito. $\mathrm{O}$ valor em regime é bastante abaixo de um típico valor de saturação de 955RPM, conforme descrito em Sidi (1997). A aceleração angular do satélite em direção inversa à da roda é visível, alcançando velocidade angular pouco abaixo de -10RPM.

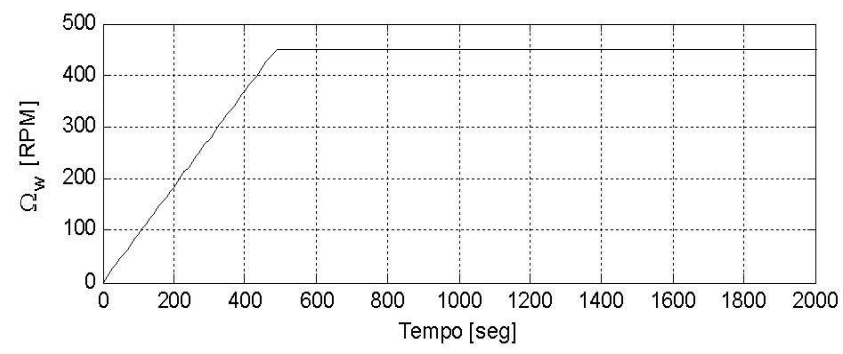

Figura 7: Velocidade angular da roda de momentum na Fase I.

A Fig.9 ilustra a convergência do erro angular de apontamento do momentum angular na Fase II, atendendo o requisito de erro angular de apontamento inferior a

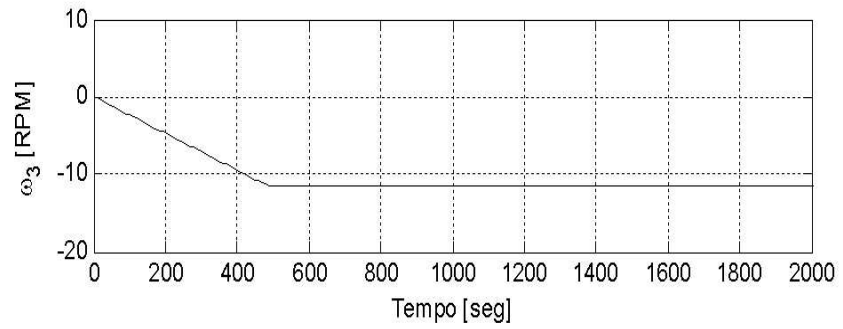

Figura 8: Componente da velocidade angular do satélite em torno do eixo de rotação da roda na Fase I.

$10^{\circ}$. Partindo de um erro inicial de $180^{\circ}$, o erro angular de apontamento é reduzido a $10^{\circ} \mathrm{em}$ aproximadamente um dia e meio e é praticamente eliminado em cerca de dois dias, permanecendo sua magnitude abaixo de $0,1^{\circ}$ em regime estacionário, mesmo com o satélite sujeito aos torques de perturbação ao longo dos dias. Isso indica a efetividade dos MTQs para gerar torque externo e alterar o momentum angular do sistema composto pelo corpo do satélite e a roda de momentum, resultando na aquisição e manutenção da atitude de referência mesmo sob as perturbações consideradas.

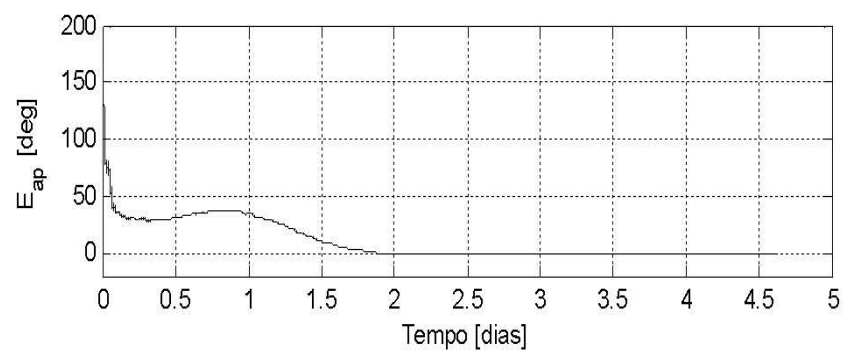

Figura 9: Erro de apontamento do momentum angular.

A taxa de convergência do estimador de bias de magnetômetro depende da intensidade do capotamento após a separação do satélite e pode durar até $12 \mathrm{~h}$ na órbita polar (Viegas, 2010; Santos, 2008). Os resultados aqui expostos empregaram as estimativas de atitude e velocidade angular realizadas após a calibração do bias de magnetômetro, conforme descrito na Seção 3.1 .

Entretanto, resultados recentes indicaram que o controle de atitude pode ser iniciado logo após a separação, usando as medidas brutas do magnetômetro para estimar a atitude e a velocidade angular do satélite. Isso se deve à aquisição de atitude pelo satélite durar bastante mais tempo que a convergência do estimador de bias do magnetômetro. Após terem convergido, as estimativas de bias são usadas para calibrar as medidas do magnetômetro e continuar o controle 
magnético com a Fase II já em andamento. Nesse caso, o tempo total de apontamento reduziu-se em cerca de 9 horas.

Os ângulos solares, mostrados na Fig.10, após oscilarem, são quase eliminados em menos de dois dias, mantendo-se abaixo de $0,1^{\circ}$ em regime estacionário, indicando apontamento do painel solar em direção do Sol.
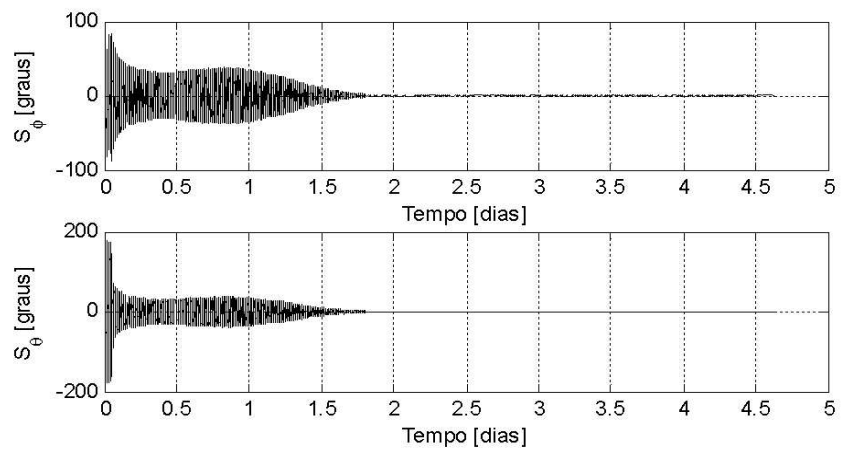

Figura 10: Ângulos solares.

A Fig.11 mostra a eficácia do amortecimento ativo de nutação resultante do controle magnético. A Fig.12 ilustra o controle efetivo em 3 eixos da velocidade angular do satélite pelos MTQs. Fica notório o amortecimento bem sucedido dos componentes de velocidade angular que são transversais ao eixo $\boldsymbol{k}_{b}$ e que causam a nutação. $\mathrm{O}$ componente de velocidade angular ao longo de $\boldsymbol{k}_{b}$ é praticamente eliminado na Fase III.

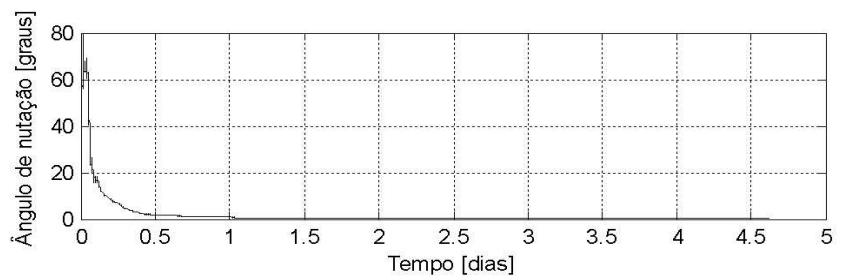

Figura 11: Ângulo de nutação.

Finalizada a Fase II após cerca de dois dias, o controle da atitude na Fase III é ilustrado na Fig.13. O controle é efetuado mediante torqueamento do motor da roda de momentum, cujo eixo já se encontra alinhado com a direção do Sol nessa fase.
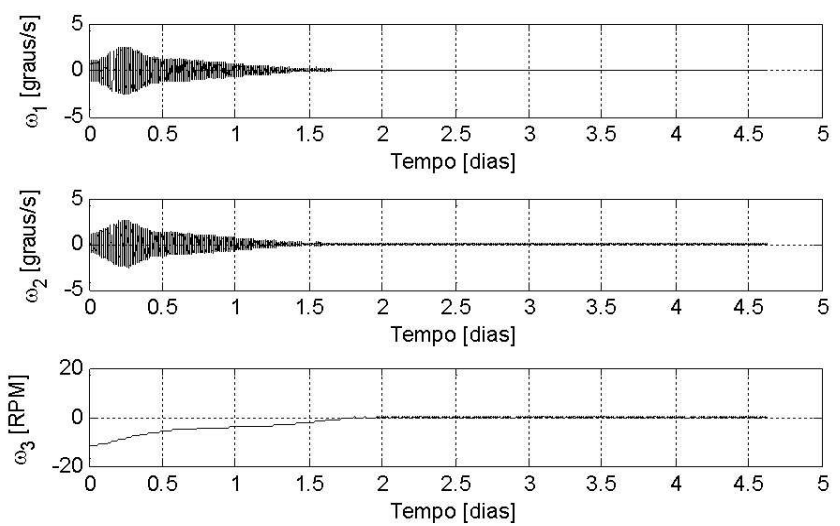

Figura 12: Velocidade angular do satélite.

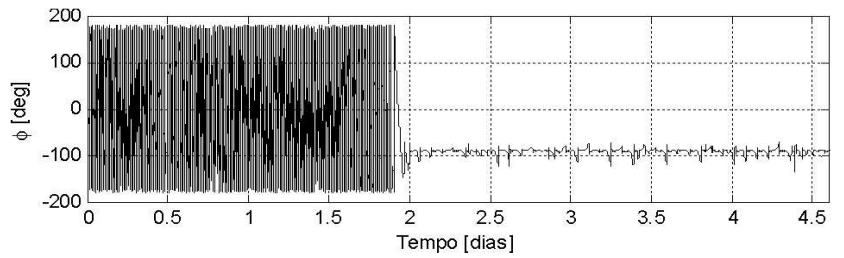

Figura 13: Atitude em torno do eixo de rotação da roda.

O período da órbita é de cerca de 100 minutos e em cada órbita ocorre um eclipse que dura em torno de 35 minutos. Como o FKE emprega as medidas do sensor de Sol na estimação de atitude e velocidade angular, resulta um erro de estimação de atitude com magnitude elevada durante os eclipses quando o sensor de Sol não está operacional. O controle é, portanto, interrompido nos eclipses e o satélite mantém sua orientação graças ao momentum angular armazenado na roda.

A oscilação da atitude em torno do eixo de rotação da roda após os cerca de dois dias requeridos para a aquisição da atitude, mostrada na Fig.13, apresenta picos que perduram por alguns instantes após o término de cada eclipse. Os piores picos apresentam magnitude em torno de $30^{\circ}$. Porém, tais erros são aceitáveis e não prejudicam a missão do satélite por causa do diagrama de irradiação das antenas embarcadas empregadas nos enlaces de telemetria, telecomando e de dados meteorológicos (Gentina et al.,2009).

A Fig.14 mostra que o torque do motor da roda emprega $0,02 \mathrm{~N} \cdot \mathrm{m}$ de magnitude na Fase I ao acelerar a roda, mantém-se inativo durante a Fase II de controle magnético durante o apontamento de seu eixo em direção ao Sol e permanece abaixo de $0,005 \mathrm{~N} \cdot \mathrm{m}$ na Fase III, sendo que o torque máximo disponível no motor é de $0,2 \mathrm{~N} \cdot \mathrm{m}$. 


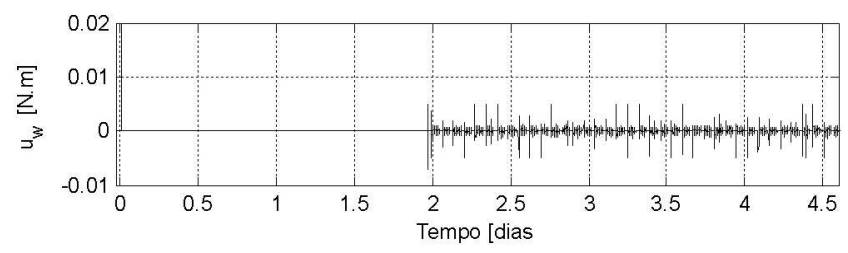

Figura 14: Torque do motor da roda de momentum.

A demanda por carga elétrica em cada um dos MTQs é mostrada na Fig.15. Observa-se uma demanda de carga, sem considerar o acionador (driver) de corrente e o magnetômetro, em torno de $2 \mathrm{~A} \cdot \mathrm{h}$ após um dia e meio de operação do triedro de MTQs, o que é seguramente viável de ser suprido pelas baterias embarcadas no satélite antes de iniciar a recarga com a energia gerada pelos painéis solares.

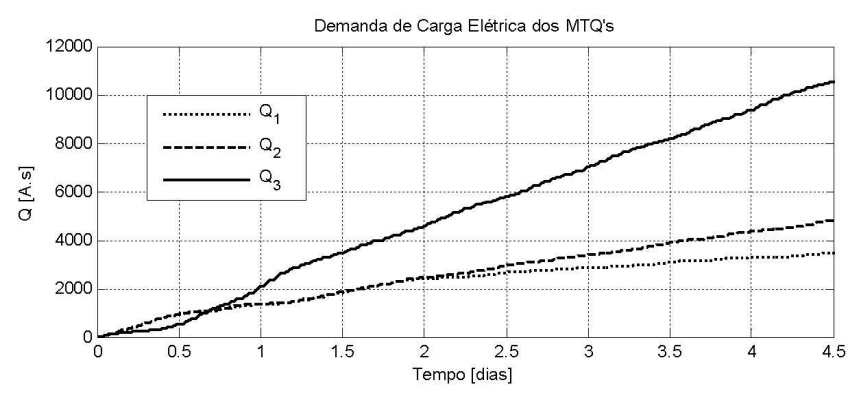

Figura 15: Demanda de carga elétrica pelo MTQ's.

\subsection{ANÁLISE DAS CONFIGURAÇÕES}

A Tab.2 ilustra algumas características de desempenho apresentadas na Revisão Preliminar de Requisitos (PRR) comparando a configuração de corpo rígido estabilizado por rotação spin investigada em Waschburger et al. (2009) com a do girostato aqui investigado.

Análise da equipe de sistema térmico indicou que a configuração aqui investigada apresentaria superaquecimento atrás da face voltada para o Sol e resfriamento excessivo na face oposta, onde baterias deveriam ficar posicionadas para obtenção de uma matriz de inércia propícia à estabilidade dinâmica. De forma a manter a posição do centro de massa e os momentos de inércia adequados ao desempenho do SCA, e considerando as dimensões e o volume limitados do satélite e os seus requisitos estruturais, o reposicionamento de equipamento se mostrou problemático. A alternativa de controle passivo de temperatura empregando tubos com fluido para dissipação térmica por convecção e radiação (heat pipes) foi considerada de complexidade significativa durante a PRR, potencialmente envolvendo um risco tecnológico que, na ocasião, foi julgado ser relevante.
A roda de momentum também foi considerada item que introduz significativa complexidade por se tratar de peça móvel que requer eletrônica adicional para acionamento do motor e lubrificante que mantenha suas características em ampla faixa de variação de temperatura.

Por isso, a configuração aqui investigada foi desconsiderada para o ITASAT-1. A PRR produziu a recomendação que o projeto do satélite fosse mantido com baixo risco tecnológico, conforme a configuração descrita em Waschburger et al. (2009), inspirada nos SCDs, mas sem empregar anel de amortecimento de nutação com fluido. O sistema de controle concebido para tal configuração, entretanto, não atende o requisito de apontamento ao operar em órbita polar, ou em órbita baixa partindo da condição inicial desfavorável cuja análise foi solicitada durante a Revisão de Definição da Missão (MDR) e que foi empregada aqui.

Portanto, a possibilidade de condição desfavorável logo após a separação em órbita polar demanda investigação adicional e revisão do SCA descrito por Waschburger et al. (2009) que foi selecionado na PRR. O uso de amortecimento passivo, sem envolver uso de fluido, foi investigado na configuração girostato em órbita polar (Almeida et al., 2010) e pode ser adaptado para emprego na configuração de corpo rígido estabilizada por spin.

\section{CONCLUSÃO}

A concepção de um satélite universitário de baixo custo busca atender as restrições de seu lançamento como carga secundária. Para atender as sugestões resultantes da Revisão de Definição da Missão (MDR), configurações distintas foram investigadas para estimar o impacto da separação do satélite em condição inicial desfavorável sobre o desempenho do Sistema de Controle de Atitude (SCA) e demais sistemas embarcados. A fase de aquisição da atitude com apontamento adequado dos painéis solares em tempo hábil é fundamental para recarregar as baterias embarcadas que, após a separação, alimentam os sistemas embarcados envolvidos nessa fase.

A configuração girostato, aqui investigada, é controlada em três eixos com uma única face voltada para o Sol. Foi investigado o desempenho do girostato partindo do pior caso de erro de apontamento inicial composto com movimento de capotamento e o acionamento da roda de momentum em seguida à separação do lançador.

O SCA é dotado de autonomia para que inicie sua ação a partir da separação e operando em malha fechada com estimativas da atitude e da velocidade angular em três eixos do satélite. Essas estimativas são fornecidas por um filtro 
Tabela 2: Análise comparativa das configurações apresentada na Revisão Preliminar de Requisitos (PRR).

\begin{tabular}{|c|c|c|}
\hline & $\begin{array}{l}\text { Estabil. rotação } \\
\text { spin }\end{array}$ & Girostato \\
\hline $\begin{array}{l}\text { Potencial para } \\
\text { geração de potência }\end{array}$ & Menor & Maior \\
\hline $\begin{array}{l}\text { Consumo instantâneo } \\
\text { do SCA (1) }\end{array}$ & $<3 \mathrm{~W}$ & $5 \mathrm{~W}-15 \mathrm{~W}$ \\
\hline Tecnologia & Mais simples & Mais complexa \\
\hline $\begin{array}{l}\text { Pontos de alta } \\
\text { temperatura }\end{array}$ & Não há & Há \\
\hline $\begin{array}{l}\text { Controle térmico } \\
\text { requerido }\end{array}$ & Passivo & $\begin{array}{l}\text { Pode requerer } \\
\text { controle ativo }\end{array}$ \\
\hline Confi abilidade & $\begin{array}{l}\text { Considerada } \\
\text { signifi cativa }\end{array}$ & $\begin{array}{l}\text { Considerada } \\
\text { duvidosa }\end{array}$ \\
\hline SDA & FKE & FKE \\
\hline Sensores & $\begin{array}{l}\text { Sensores de Sol } \\
\text { e magnetômetro }\end{array}$ & $\begin{array}{l}\text { Sensores de Sol } \\
\text { e magnetômetro }\end{array}$ \\
\hline Atuadores & $\begin{array}{l}\text { MTQs com } \\
\text { núcleo de ar }\end{array}$ & $\begin{array}{ll}\text { MTQs } & \text { com } \\
\text { núcleo de } & \text { ar e } \\
\text { roda } & \end{array}$ \\
\hline Peso ${ }^{(1)}$ & 3 MTQs & $\begin{array}{l}3 \text { MTQs }+1 \\
\text { roda }(4)\end{array}$ \\
\hline $\begin{array}{l}\text { Estabilização na fase } \\
\text { operacional }\end{array}$ & $\begin{array}{l}2 \quad \text { eixos } \quad \mathrm{e} \\
\text { velocidade } \\
\text { spin }\end{array}$ & 3 eixos \\
\hline $\begin{array}{ll}\text { Acurácia } & \text { do } \\
\text { apontamento } & \\
\text { (simulação) } & \end{array}$ & Em torno de $5^{\circ}$ & Melhor que $5^{\circ}$ \\
\hline $\begin{array}{l}\text { Duração da manobra } \\
\text { de aquisição }\end{array}$ & 5 dias $(6)$ & $2 \operatorname{dias}(6)$ \\
\hline Rigidez giroscópica & $\begin{array}{l}\text { Maior } \\
(20 \mathrm{~N} \cdot \mathrm{m} \cdot \mathrm{s})\end{array}$ & $\begin{array}{l}\text { Menor } \\
(10 \mathrm{~N} \cdot \mathrm{m} \cdot \mathrm{s})\end{array}$ \\
\hline $\begin{array}{l}\text { Manobrabilidade do } \\
\text { eixo de } \operatorname{spin}\end{array}$ & Menor & Maior \\
\hline $\begin{array}{l}\text { Capacidade } \\
\text { para plataforma } \\
\text { multimissão }\end{array}$ & Não & Sim \\
\hline $\begin{array}{l}\text { Geração de novo } \\
\text { conhecimento }\end{array}$ & Sim & Certamente \\
\hline
\end{tabular}

(1) Desconsidera o acionamento por corrente dos MTQs e o magnetômetro.

(2) Considerando experiência prévia com os Satélites de Coleta de Dados 1 e 2 (SCDs), lançados como cargas pagas com condição inicial controlada para reduzir dispersão da atitude do eixo de spin após a separação, característica que não está prevista para o satélite universitário de baixo custo.

(3) Após aquisição de atitude, ocorrência de falha de travamento da roda resulta em estabilização por spin com eixo apontando para o Sol e irradiação adequada sobre o painel solar por um período de cerca de um mês. Entretanto, essa confi guração, autônoma, ainda não foi realizada no Brasil e lançada com sucesso.

(4) Roda de momentum com massa de $5 \mathrm{~kg}$ para armazenar momentum angular de $10 \mathrm{~N} \cdot \mathrm{m} \cdot \mathrm{s}$ (Larsen e Wertz, 1999).

(5) Considerando satélite separando-se de lançador com seu último estágio controlado em 3 eixos.

(6) Após o bias de magnetômetro ter sido estimado e calibrado, o que pode durar de 1 hora até 12 horas, e o campo geomagnético sendo medido com período de amostragem, respectivamente, de $1 \mathrm{~s}$ ou $10 \mathrm{~s}$ dependendo de quão intenso é o movimento inicial de capotamento do satélite (Santos et al., 2009). de Kalman estendido (FKE) que processa medidas vetoriais da direção do Sol e do campo geomagnético oriundas, respectivamente, de sensores de Sol e magnetômetro. O controle de atitude é interrompido durante os eclipses, quando o erro de estimação diverge devido à perda das medidas dos sensores de Sol. A rigidez giroscópica devido ao momentum angular da roda mantém o satélite orientado de forma adequada até ressurgir da sombra da Terra.

Partindo de um erro angular de apontamento inicial de $180^{\circ}$ e capotando lentamente, os resultados mostraram a eficácia do SCA no apontamento do satélite ao requerer aproximadamente um dia e meio para prover um erro de apontamento com magnitude inferior a $10^{\circ}$ e cerca de dois 2 dias para manter a magnitude do erro abaixo de $0,1^{\circ}$.

A duração das manobras em relação à capacidade de carga das baterias embarcadas confirmou a viabilidade da configuração girostato. Entretanto, devido à complexidade advinda do emprego da roda e do controle térmico necessário à distribuição adequada de temperatura ao ser mantida uma mesma face continuamente exposta ao Sol, a Revisão Preliminar de Requisitos (PRR) recomendou a configuração de corpo rígido estabilizado por rotação spin, que pode empregar controle passivo de temperatura, mas cuja aquisição de atitude sob condição inicial desfavorável em órbita polar demanda investigação adicional para alcançar desempenho aceitável.

Está em andamento a implementação em tempo real do SCA na configuração selecionada pela PRR. A implementação emprega o kit Atmel TSC695F de desenvolvimento de computador bordo (Atmel, 2011) robustecido contra radiação para aplicação espacial. Apresenta processador de 32 bits, arquitetura SPARC, clock de $25 \mathrm{MHz}$, desempenho de 20MIPS e 5 MFLOPS, 32MB de memória RAM e $15 \mathrm{MB}$ de PROM. A implementação emprega o sistema operacional de tempo real RTEMS e está sendo integrada em um simulador hardware-in-the-loop da dinâmica de atitude do satélite (dos Santos e Waldmann, 2011).

Ambas as configurações, a de corpo rígido estabilizada por rotação spin e a girostato, continuam sendo investigadas no projeto FINEP/DCTA/INPE Sistemas Inerciais para Aplicação Aeroespacial (SIA), que elenca entre seus objetivos o desenvolvimento de algoritmos para estimação e controle de atitude em 3 eixos para satélites, e no qual o ITA participa com foco na formação de quadros para atuar na área de engenharia inercial.

\section{AGRADECIMENTOS}

Os autores agradecem as valiosas discussões técnicas e comunicações pessoais realizadas com os pesquisadores 
do INPE Dr. Helio Koiti Kuga, Dr. Valdemir Carrara, Dr. Roberto Vieira da Fonseca Lopes, Dr. Sebastião Varotto Corsatto e Dr. Wilson Yamaguti. São aqui reconhecidos o suporte financeiro concedido por meio de bolsa de estudos do projeto AEB/INPE/ITA/ITASAT da Agência Espacial Brasileira aos três primeiros autores, assim como o apoio logístico e equipamentos adquiridos pelo projeto FINEP/DCTA/INPE Sistemas Inerciais para Aplicação Aeroespacial (SIA).

Os autores também agradecem os comentários dos revisores, que muito contribuíram para a maior clareza do artigo.

\section{REFERÊNCIAS}

Alfriend, K. T. (1974). Partially Filled Viscous Ring Nutation Damper. Journal of Spacecraft, Vol.11, no.7, pp.456-462.

Almeida, P. de S. L.; Viegas, W. da V. C. e Waldmann, J. (2010). Controle de Atitude do Satélite Universitário ITASAT Estabilizado por Rotação Dual para Avaliação de Amortecimento Mecânico de Nutação. XVIII Congresso Brasileiro de Automática, Bonito, MS.

Alonso, R. e Shuster, M. D. (2002). TWOSTEP: A Fast Robust Algorithm for Atitude-Independent Magnetometer Bias Determination. The Journal of the Astronautical Sciences, Vol. 50, No. 4, pp. 433-451.

Atmel (2011). TSC695F SPARC 32-bit Space Processor User Manual. Documento 4148H-AERO-12/03. www.atmel.com/dyn/resources/prod_documents/doc4148.pdf. Último acesso em 6 de março de 2011.

Carrara, V. e Guedes, U. T. V. (1994). Attitude Control Aspects for SCD1 and SCD2. RBCM - J. of the Brazilian Soc. Mechanical Sciences, vol. XVI, pp. 83-87.

Carrara, V. (1996). Projeto de Bobinas Magnéticas para Uso em Satélites. Congresso Brasileiro de Eletromagnetismo CBMAG 96, Ouro Preto, MG.

Chang, Y.-K., Lee, B.-H. e Kim, S.-J. (2006). Momentum Wheel Start-Up Method for HAUSAT-2 Ultra-Small Satellite. Aerospace Science and Technology, Vol. 10, pp. 168-174.

Crassidis, J. L.; Lai, K.-L. e Harman, R. R. (2005). Real-Time Attitude-Independent Three-Axis Magnetometer Calibration. Journal of Guidance, Control, and Dynamics, Vol. 28, No. 1, pp. 115-120.

da Fonseca, I. M. e de Souza, P.N. (1990). Modelagem do Movimento Rotacional de um Corpo Rígido Dotado de Um Amortecedor de Nutação Anular Viscoso e Sua
Validação Experimental. RBCM - J. of the Brazilian Soc. Mechanical Sciences, vol. XII, no. 1, pp. 89-111.

dos Santos, S. R. G. e Waldmann, J. (2011). Real-Time Implementation of Low-Cost University Satellite 3-Axis Attitude Determination and Control System. Proceedings of the XXIBrazilian Congress of Mechanical Engineering - COBEM 2011, Natal, $\mathrm{RN}$, Brasil. (submetido)

Ergin, E. I. e Wheeler, P. C. (1965). Magnetic Attitude Control of a Spinning Satellite. J. Spacecraft, Vol. 2 No. 6, pp. 646-850.

Gentina, J.; Yamaguti, W.; Varotto, S. E. C.; Waldmann, J. e Fernandes, D. (2009). A Proposal for ITASAT Satellite Configuration And Its Preliminary Mission Analysis. Brazilian Symposium on Aerospace Eng. \& Applications, São José dos Campos - SP.

Godoi, R. G. e Waldmann, J. (2010). Simulação da Implementação Embarcada do Sistema de Controle de Atitude de Satélite Universitário de Baixo Custo. Anais do VI Simpósio Brasileiro de Engenharia Inercial, Rio de Janeiro, RJ.

Gravdahl, J. T. (2004). Magnetic Attitude Control for Satellites. Proceedings of the $43^{\text {rd }}$ IEEE Conference on Decision and Control, Atlantis, Paradise Island, Bahamas, pp.261-266.

Hughes, P. C. (1986). Spacecraft Attitude Dynamics. John Wiley and Sons.

Kuga, H. K.; Ferreira, L. D. D. e Guedes, U. T. V. (1987). Simulação de Atitude e de Manobras para o Satélite Brasileiro Estabilizado por Rotação, Instituto Nacional de Pesquisas Espaciais, São José dos Campos - SP, Brasil.

Kuga, H. K.; Orlando, V. e Lopes, R. V. F. (1999). Flight Dynamics Operations During LEOP for INPE's Second Environmental Data Collecting Satellite SCD2. RBCM - J. of the Brazilian Soc. Mechanical Sciences, vol. XXI, Special Issue, pp. 339-344.

Kulkarni, J. e Campbell, M. (2004). An Approach to Magnetic Torque Attitude Control Via H-infinity Control for LTV Systems. Proceedings of the $43^{\text {rd }}$ IEEE Conference on Decision and Control, Atlantis, Paradise Island, Bahamas, pp.273-276.

Larson, W.J. e Wertz, J. R. (eds.). (1999). Space Mission Analysis and Design. $3^{a}$ edição, Microcosm Press.

Lopes, R. V. da F. (1987). Modelagem de Um Amortecedor de Nutação para Satélites Estabilizados por Rotação. IX Congresso Brasileiro de Engenharia Mecânica, Florianópolis, SC, pp.761-764. 
Lopes, R. V. da F. L.; Orlando, V.; Kuga, H. K., Guedes, U. T. V. e Rao, K. R. (1994). Attitude Determination of the Brazilian Satellite SCD1. RBCM - J. of the Brazilian Soc. Mechanical Sciences, vol. XVI, Special Issue, pp. 11-18.

Lovera, M., De Marchi, E., e Bittanti, S. (2002). Periodic Attitude Control Techniques for Small Sattelites With Magnetic Actuators. IEEE Transactions on Control Systems Technology, Vol. 10, No. 1, pp. 90-95.

McLean, S.; MacMillan, S.; Maus, S.; Lesur, V. e Dater, D. (2004). US/UK World Magnetic Model for 2005-2010, Technical Report NESDIS/NGDC-1.

Mesch, F. (1969). Magnetic Components for the Attitude Control of Space Vehicles. IEEE Transactions on Magnetics, Vol. 5, No. 3, pp. 586-592.

Rennard, M. L. (1967). Command Laws for Magnetic Attitude Control of Spin-Stabilized Earth Satellites. J. Spacecraft, Vol. 4, No. 2, pp. 156-163.

Roth, Y. (2001). Magnetotorquer for CNES/ $\mu$ SAT Electrical Interface for Control Document. Relatório $\mu$ ST-CI-S-4-236-CNES, edição (issue) 5, rev. 0, 02 de julho de 2001.

Santos, D.A. (2008). Estimação de Atitude e Velocidade Angular de Satélites Utilizando Medidas do Campo Geomagnético e da Direção do Sol. Dissertação de mestrado - Instituto Tecnológico de Aeronáutica - ITA, São José dos Campos - SP.

Santos, D. A. e Waldmann, J. (2009). Attitude and Angular Rate Estimation from Vector Measurements of Magnetometer and Sun Sensors for a Low-Cost Satellite. Proceedings of the $X X^{\text {th }}$ Congresso Internacional de Engenharia Mecânica - COBEM 2009, Gramado, RS, Brasil.

Shigehara, M. (1972). Geomagnetic Attitude Control of an Axisymmetric Spinning Satellite, Journal of Spacecraft, Vol. 9, No. 6, pp. 391-398.

Sidi, J. M. (1997). Spacecraft Dynamics \& Control - A Practical Engineering Approach, Cambridge University, Cambridge, UK.

Sorensen, J. A. (1971). A Magnetic Attitude Control System for an Axisymmetric Spinning Spacecraft, J. Spacecraft, Vol. 8, No. 5, pp. 441-455.

Vallado, D. A.; Crawford, P.; Hujsak, R. e Kelso, T. S. (2006). Revisiting Spacecraft Report \#3, American Institute of Aeronautics and Astronautics - AIAA, Astrodynamics Specialist Conference and Exhibit, Keystone, Colorado, USA.
Viegas, W. da V. C. (2010). Sistemas de Determinação e Controle Autônomos de Atitude para Satélites de Baixo Custo Estabilizados por Roda de Momentum e Atuação Magnética com Sensores de Sol e Magnetômetro. Dissertação de mestrado, ITA, São José dos Campos, SP.

Viegas, W. da V. C. e Waldmann, J. (2010). Aquisição Inicial e Estabilização de Atitude em 3 Eixos de Um Satélie Universitário de Baixo Custo por Viés em Roda de Momentum e Controle Magnético. Anais do VI Congresso Brasileiro de Engenharia Mecânica CONEM 2010, Campina Grande, PB.

Vreeburg, J. P. B. (2005). Spacecraft Maneuvers and Slosh Control. IEEE Control Systems Magazine, pp.12-16.

Waschburger, R.; Santos, D. A. e Waldmann, J. (2009). Magnetotorquer-Only Attitude Control System Robust to Wide Range of Initial Conditions for Low-Cost Spin Stabilized ITASAT Satellite, Proceedings of the $X X^{\text {th }}$ Congresso Internacional de Engenharia Mecânica COBEM 2009, Gramado - RS, Brasil.

Wertz, J. R. (ed.). (1978). Spacecraft Attitude Determination and Control, The Netherlands: Kluwer Academic Publishers.

Wheeler, C. P. (1967). Spinning Spacecraft Attitude Control Via the Environmental Magnetic Field. J. Spacecraft, Vol. 4, No. 12, pp. 1631-1637.

Zhao, S. (2009). Quadratic Stabilization for a Class of Switched Nonlinear Singular Systems. International Journal of Information and Systems Sciences, Vol. 5, N. 3-4, pp. 425-429. 